\title{
Synthesis of Polyfunctionalized Amino Furans with Long Conjugated Aromatic Systems Using Nucleophilic Aromatic Isocyanide
}

\author{
MOHAMMAD QANDALEE ${ }^{\mathrm{a}^{*}}$, MASOUD MOKHTARY $^{\mathrm{b}}$, \\ SAKINEH ASGHARI ${ }^{\mathrm{c}}$, and MEHRDAD MEHRZADI ${ }^{\mathrm{d}}$ \\ ${ }^{a}$ Department of biology, Garmsar Branch \\ Islamic Azad University, Garmsar, Iran \\ ${ }^{b}$ Department of Chemistry, Rasht Branch \\ Islamic Azad University, PO Box 41325-3516, Rasht, Iran \\ ${ }^{c}$ Department of Organic Chemistry, Faculty of Chemistry, \\ University of Mazandaran, Babolsar, 47415, Iran \\ ${ }^{\mathrm{d}}$ Department of Chemistry, Ayatollah Amoli Branch, \\ Islamic Azad University, Amol, Iran \\ qandalee@gmail.com
}

Received 25 October 2011; Accepted 29 December 2011

\begin{abstract}
Polyfunctionalized amino furans with long conjugated aromatic systems have been synthesized by three component reaction of aldehydes, acetylenic esters and aromatic nucleophilic isocyanide.
\end{abstract}

Keywords: 2,6-Dimethylphenyl isocyanide, Acetylenic esters, Aldehydes, Amino furans.

\section{Introduction}

Polysubstituted furans are key structures in a lot of naturally occurring products and pharmaceutical compounds ${ }^{1}$. Because of this importance, synthesis of such compounds attracted great attentions. The nucleophilic character of isocyanide with a formally divalent carbon atom has made it as one of the compounds with an extraordinary functional group which is very important species in the synthesis of heterocycles like furans ${ }^{2,3}$. Recently, we considered the chemiluminescene properties of polyfunctionalized conjugated furans ${ }^{4,5}$. In this work we have used aromatic isocyanide to synthesize new furans with longer aromatic conjugated systems. The structure shows that three aromatic rings are attached by amine and olefin groups. Therefore, we expect higher chemiluminescence activities in these compounds (Scheme1). 


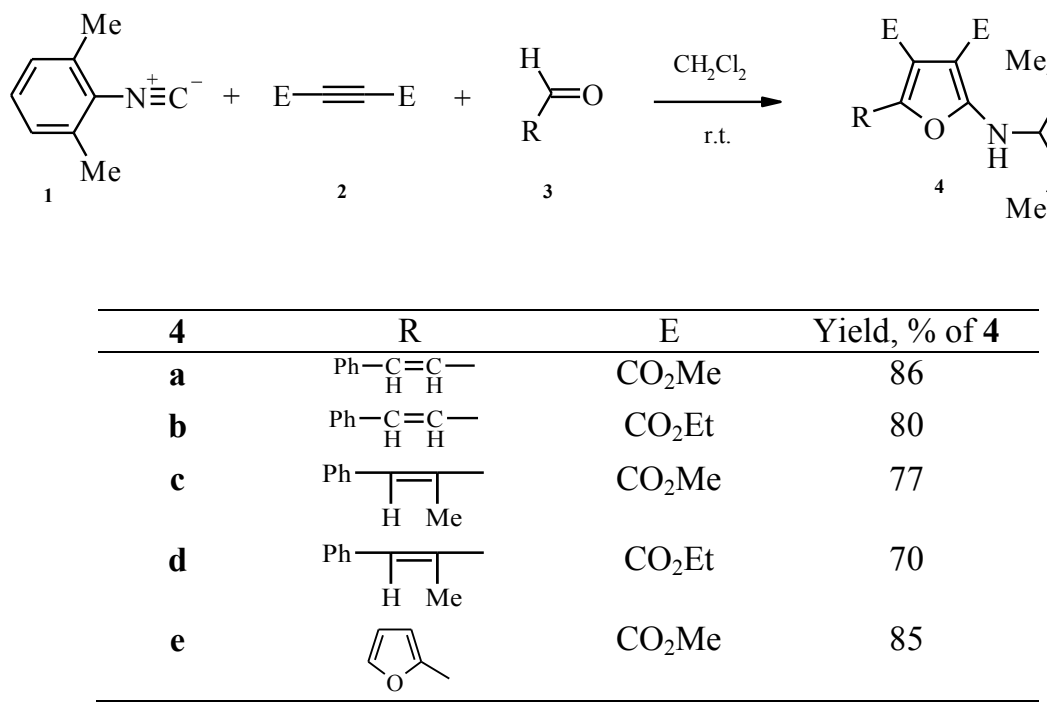

Scheme 1. Synthesis of compound 4.

\section{Experimental}

Dialkyl aceylenedicarboxylates, alkylisocyanide and cinnamaldehyde were purchased from fluka (Buchs, Switzerland) and were used without further purification. Melting points were measured on a thermo scientific apparatus. ${ }^{1} \mathrm{H}$ and ${ }^{13} \mathrm{C}$ NMR spectra were measured with a Bruker DRX-400 Avance spectrometer at 400 and $100.6 \mathrm{MHz}$, respectively. Mass spectra were recorded on a Agilent Technology (HP) 5973 mass spectrometer operating at an ionization potential of $70 \mathrm{eV}$. IR spectra were recorded on a FT-IR Bruker (WQF-510) spectrometer.

\section{General procedure for the preparation of compound 4}

To a magnetically stirred solution of cinnamaldehyde $(2 \mathrm{mmol})$ and dialkyl acetylenedicarboxylate $(2 \mathrm{mmol})$ in $\mathrm{CH}_{2} \mathrm{Cl}_{2}(10 \mathrm{~mL})$, dropwise, $2 \mathrm{mmol}$ of alkyl isocyanide in $\mathrm{CH}_{2} \mathrm{Cl}_{2}$ $(4 \mathrm{~mL})$ was added at room temperature. The mixture was stirred at room temperature for $24 \mathrm{~h}$. The solvent was removed under reduced pressure and the residue was purified by silica gel (Merck silica gel, 230-240 mesh) column chromatography using hexane:ethyl acetate $(80: 20)$ to afford pure products.

\section{Results and Discussion}

On the basis of the extraordinary properties of isocyanide ${ }^{6-17}$, it is reasonable to assume that the reaction starts by Micheal addition of isocyanide to the acetylenic ester to form the zwitterionic intermediate 5, which attacks the carbonyl group of the aldehyde $\mathbf{3}$. Then, nucleophilic addition to the nitrile iminium moiety leads to the formation of the fivemembered ring 6 which is aromatized to functionalized furan 4 by [1,5]-H shift (Scheme 2). 


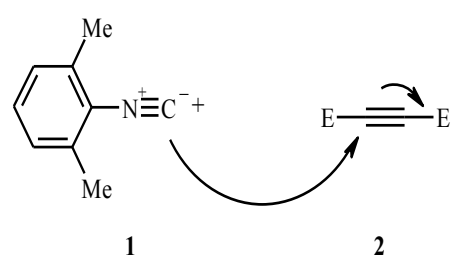

2

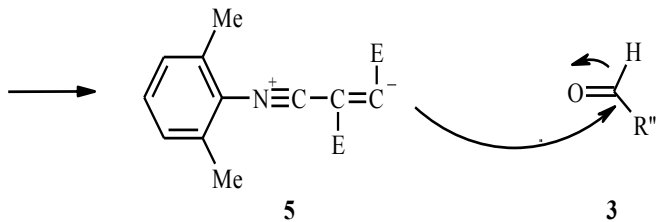

3
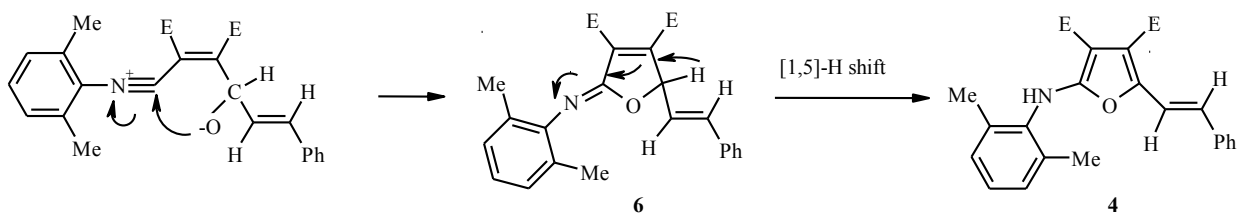

Scheme 2. Proposed mechanism for the formation of compound 4.

\section{Spectroscopic data}

Dimethyl 2-(2,6-Dimethyl-phenylamino)-5-[(E)-2-phenyl-1-ethenyl]-3,4-furan dicarboxylate (4a)

Yellow powder, m.p. $117-119{ }^{\circ} \mathrm{C}$, yield $86 \%$, IR $(\mathrm{KBr})\left(v_{\max }, \mathrm{cm}^{-1}\right): 1662-1712(\mathrm{C}=\mathrm{O})$ and $3311(\mathrm{NH}) ;{ }^{1} \mathrm{H}$ NMR $\left(400 \mathrm{MHz}, \mathrm{CDCl}_{3}\right): \delta_{\mathrm{H}} 2.32\left(6 \mathrm{H}, \mathrm{s}, 2 \mathrm{CH}_{3}\right), 3.89$ and $3.96(6 \mathrm{H}, 2 \mathrm{~s}, 2$ $\left.\mathrm{OCH}_{3}\right), 6.67\left(1 \mathrm{H}, \mathrm{d}, \mathrm{CH}=\mathrm{CH},{ }^{3} \mathrm{~J}_{\mathrm{HH}}=16.4 \mathrm{~Hz}\right), 7.08\left(1 \mathrm{H}, \mathrm{d}, \mathrm{CH}=\mathrm{CH},{ }^{3} \mathrm{~J}_{\mathrm{HH}}=16.4 \mathrm{~Hz}\right), 7.19$ $(3 \mathrm{H}, \mathrm{m}, \mathrm{Ph}), 7.22\left(1 \mathrm{H}, \mathrm{t},{ }^{3} \mathrm{~J}_{\mathrm{HH}}=7.2 \mathrm{~Hz}, \mathrm{H}_{\mathrm{para}}\right), 7.32\left(2 \mathrm{H}, \mathrm{t},{ }^{3} \mathrm{~J}_{\mathrm{HH}}=7.2 \mathrm{~Hz}, \mathrm{H}_{\text {meta }}\right), 7.40(2 \mathrm{H}, \mathrm{d}$, $\left.{ }^{3} \mathrm{~J}_{\mathrm{HH}}=7.2 \mathrm{~Hz}, \mathrm{H}_{\text {ortho }}\right), 8.36(1 \mathrm{H}, \mathrm{s}, \mathrm{NH}) ;{ }^{13} \mathrm{C} \mathrm{NMR}\left(400 \mathrm{MHz}, \mathrm{CDCl}_{3}\right): \delta_{\mathrm{C}} 18.50\left(2 \mathrm{CH}_{3}\right), 51.4$ and $52.1\left(2 \mathrm{OCH}_{3}\right), 87.83,113.91,145.93$ and 160.62 (furan), 114.01 and $128.01(\mathrm{C}=\mathrm{C}$ alkene $), 126.59\left(\mathrm{C}_{\text {ortho }}\right), 127.23\left(\mathrm{C}_{\text {para }}\right), 128.51\left(\mathrm{C}_{\text {meta }}\right), 136.43\left(\mathrm{C}_{\text {ipso }}\right), 128.64\left(\mathrm{C}_{\text {ortho }}\right), 128.82$ $\left(\mathrm{C}_{\text {para }}\right), 134.10\left(\mathrm{C}_{\text {meta }}\right), 135.26\left(\mathrm{C}_{\text {ipso }}\right), 164.04$ and $165.44(2 \mathrm{CO}) ; \mathrm{MS}: \mathrm{m} / \mathrm{z}(\%): 59$ $\left(\mathrm{COOCH}_{3}, 7\right), 77(\mathrm{Ph}, 25), 103\left(\mathrm{PhCH}=\mathrm{CH}^{+}, 54\right), 285\left(\mathrm{M}^{+}-\left[\left(\mathrm{CH}_{3}\right)_{2} \mathrm{PhNH}^{+}\right], 32\right), 341$ $\left(\mathrm{M}^{+}-\left[2 \mathrm{OCH}_{3}+\mathrm{H}\right], 45\right), 374\left(\mathrm{M}^{+}-2 \mathrm{CH}_{3}, 78\right), 404\left(\mathrm{M}^{+}, 100\right)$, Anal. Calcd for $\mathrm{C}_{24} \mathrm{H}_{23} \mathrm{NO}_{5}$ (405.42): C, 71.10; H, 5.71; N, 3.45. Found: C, 71.17; H, 5.74; N, 3.47.

\section{Diethyl 2-(2,6-Dimethyl-phenylamino)-5-[(E)-2-phenyl-1-ethenyl]-3,4-furan dicarboxylate (4b)}

Yellow powder, m.p. $128-130{ }^{\circ} \mathrm{C}$, yield $80 \%$, IR $(\mathrm{KBr})\left(v_{\max }{ }^{\prime} \mathrm{cm}^{-1}\right): 1660-1710(\mathrm{C}=\mathrm{O})$ and $3276(\mathrm{NH}) ;{ }^{1} \mathrm{H}$ NMR $\left(400 \mathrm{MHz}, \mathrm{CDCl}_{3}\right): \delta_{\mathrm{H}} 1.39\left(3 \mathrm{H}, \mathrm{t},{ }^{3} \mathrm{~J}_{\mathrm{HH}}=7.2 \mathrm{~Hz}, \mathrm{CH}_{3}\right), 1.44(3 \mathrm{H}, \mathrm{t}$, $\left.{ }^{3} \mathrm{~J}_{\mathrm{HH}}=7.2 \mathrm{~Hz}, \mathrm{CH}_{3}\right), 2.32\left(6 \mathrm{H}, \mathrm{s}, 2 \mathrm{CH}_{3}\right), 4.35\left(2 \mathrm{H}, \mathrm{q},{ }^{3} \mathrm{~J}_{\mathrm{HH}}=7.2 \mathrm{~Hz}, \mathrm{OCH}_{2}\right), 4.42\left(2 \mathrm{H}, \mathrm{q},{ }^{3} \mathrm{~J}_{\mathrm{HH}}\right.$ $\left.=7.2 \mathrm{~Hz}, \mathrm{OCH}_{2}\right), 6.66\left(1 \mathrm{H}, \mathrm{d}, \mathrm{CH}=\mathrm{CH},{ }^{3} \mathrm{~J}_{\mathrm{HH}}=16 \mathrm{~Hz}\right), 7.08\left(1 \mathrm{H}, \mathrm{d}, \mathrm{CH}=\mathrm{CH},{ }^{3} \mathrm{~J}_{\mathrm{HH}}=16 \mathrm{~Hz}\right)$, $7.20(3 \mathrm{H}, \mathrm{m}, \mathrm{Ph}), 7.22\left(1 \mathrm{H}, \mathrm{t},{ }^{3} \mathrm{~J}_{\mathrm{HH}}=7.2 \mathrm{~Hz}, \mathrm{H}_{\text {para }}\right), 7.33\left(2 \mathrm{H}, \mathrm{t},{ }^{3} \mathrm{~J}_{\mathrm{HH}}=7.2 \mathrm{~Hz}, \mathrm{H}_{\text {meta }}\right), 7.40$ $\left(2 \mathrm{H}, \mathrm{d},{ }^{3} \mathrm{~J}_{\mathrm{HH}}=7.2 \mathrm{~Hz}, \mathrm{H}_{\mathrm{ortho}}\right), 8.38(1 \mathrm{H}, \mathrm{s}, \mathrm{NH}) ;{ }^{13} \mathrm{C} \mathrm{NMR}\left(400 \mathrm{MHz}, \mathrm{CDCl}_{3}\right): \delta_{\mathrm{C}} 14.36$ and $14.48\left(2 \mathrm{CH}_{3}\right), 18.58\left(2 \mathrm{CH}_{3} \mathrm{Ph}\right), 60.07$ and $61.15\left(2 \mathrm{OCH}_{2}\right), 88.09,113.93,145.53$ and 160.51 (furan), 114.55 and $128.38(\mathrm{C}=\mathrm{C}$ alkene $), 126.53\left(\mathrm{C}_{\text {ortho }}\right), 127.16\left(\mathrm{C}_{\text {para }}\right), 127.91$ $\left(\mathrm{C}_{\text {meta }}\right), 136.53\left(\mathrm{C}_{\text {ipso }}\right), 128.50\left(\mathrm{C}_{\text {ortho }}\right), 128.65\left(\mathrm{C}_{\text {para }}\right), 134.19\left(\mathrm{C}_{\text {meta }}\right), 135.23\left(\mathrm{C}_{\text {ipso }}\right), 163.77$ and 165.10 (2 CO); MS: $m / z(\%): 103\left(\mathrm{PhCH}=\mathrm{CH}^{+}, 59\right), 265\left(\mathrm{M}^{+}\right.$-[PhCH=CHfuran], 91), $313\left(\mathrm{M}^{+}-\left[\left(\mathrm{CH}_{3}\right)_{2} \mathrm{PhNH}_{2}+\mathrm{H}\right], 39\right), 342\left(\mathrm{M}^{+}-2 \mathrm{CH}_{3} \mathrm{CH}_{2} \mathrm{OH}, 38\right), 361\left(\mathrm{M}^{+}-\mathrm{COOCH}_{2} \mathrm{CH}_{3}\right.$, 82), $434\left(\mathrm{M}^{+}, 100\right)$, Anal. Calcd for $\mathrm{C}_{26} \mathrm{H}_{27} \mathrm{NO}_{5}$ (433.47): C, 72.04; H, 6.27; N, 3.23. Found: C, 72.10; H, 6.31; N, 3.25. 
Dimethyl 2-[(2,6-dimethylphenyl)amino]-5-[(E)-1-methyl-2-phenylvinyl]-3,4-furan dicarboxylate (4c)

Yellow powder, m.p. $125-127{ }^{\circ} \mathrm{C}$, yield $77 \%$, IR $(\mathrm{KBr})\left(v_{\max }{ }^{\mathrm{cm}^{-1}}\right): 1675-1735(\mathrm{C}=\mathrm{O})$ and $3305(\mathrm{NH}) ;{ }^{1} \mathrm{H}$ NMR $\left(400 \mathrm{MHz}, \mathrm{CDCl}_{3}\right): \delta_{\mathrm{H}} 2.02\left(3 \mathrm{H}, \mathrm{s}, \mathrm{CH}_{3}\right), 2.32\left(6 \mathrm{H}, \mathrm{s}, 2 \mathrm{CH}_{3}\right), 3.86$ and $3.93\left(6 \mathrm{H}, 2 \mathrm{~s}, 2 \mathrm{OCH}_{3}\right), 6.67(1 \mathrm{H}, \mathrm{s}, \mathrm{CH}=\mathrm{CH}), 7.15(3 \mathrm{H}, \mathrm{m}, \mathrm{Ph}), 7.22\left(1 \mathrm{H}, \mathrm{t},{ }^{3} \mathrm{~J}_{\mathrm{HH}}=7.6 \mathrm{~Hz}\right.$, $\left.\mathrm{H}_{\text {para }}\right), 7.32\left(2 \mathrm{H}, \mathrm{t},{ }^{3} \mathrm{~J}_{\mathrm{HH}}=7.6 \mathrm{~Hz}, \mathrm{H}_{\text {meta }}\right), 7.34\left(2 \mathrm{H}, \mathrm{d},{ }^{3} \mathrm{~J}_{\mathrm{HH}}=7.6 \mathrm{~Hz}, \mathrm{H}_{\text {ortho }}\right), 8.07(1 \mathrm{H}, \mathrm{s}, \mathrm{NH})$; ${ }^{13} \mathrm{C}$ NMR $\left(400 \mathrm{MHz}, \mathrm{CDCl}_{3}\right): \delta_{\mathrm{C}} 14.27\left(\mathrm{CH}_{3}\right), 18.49\left(2 \mathrm{CH}_{3}\right), 51.47$ and $52.71\left(2 \mathrm{OCH}_{3}\right)$, 88.56, 113.80, 142.90 and 159.30 (furan), 124.84 and $128.14\left(\mathrm{C}=\mathrm{C}\right.$ alkene), $126.16\left(\mathrm{C}_{\text {ortho }}\right)$, $126.78\left(\mathrm{C}_{\text {para }}\right), 127.19\left(\mathrm{C}_{\text {meta }}\right), 136.91\left(\mathrm{C}_{\text {ipso }}\right), 128.46\left(\mathrm{C}_{\text {ortho }}\right), 129.23\left(\mathrm{C}_{\text {para }}\right), 134.10\left(\mathrm{C}_{\text {meta }}\right)$, $135.23\left(\mathrm{C}_{\text {ipso }}\right), 164.86$ and $166.20(2 \mathrm{CO})$; MS: $\mathrm{m} / \mathrm{z}(\%): 59\left(\mathrm{COOCH}_{3}, 7\right), 121\left(\left(\mathrm{CH}_{3}\right)_{2}\right.$ $\left.\mathrm{PhNH}_{2}, 82\right), 267\left(\mathrm{M}^{+}-\left[2 \mathrm{CH}_{3} \mathrm{COOH}+\mathrm{HOCH}_{3}\right], 25\right), 328\left(\mathrm{M}^{+}-\left[2 \mathrm{CH}_{4}+\mathrm{COOCH}_{3}\right], 34\right)$, $388\left(\mathrm{M}^{+}-\mathrm{OCH}_{3}, 54\right), 419\left(\mathrm{M}^{+}, 100\right)$, Anal. Calcd for $\mathrm{C}_{25} \mathrm{H}_{25} \mathrm{NO}_{5}$ (419.45): C, 71.58; H, 6.00; $\mathrm{N}, 3.33$. Found: C, 71.64; H, 6.04; N, 3.34.

\section{Diethyl 2-[(2,6-dimethylphenyl)amino]-5-[(E)-1-methyl-2-phenylvinyl]-3,4-furan dicarboxylate (4d)}

Yellow powder, m.p. $126-128{ }^{\circ} \mathrm{C}$, yield $70 \%$, IR $(\mathrm{KBr})\left(v_{\max } \mathrm{cm}^{-1}\right): 1675-1739(\mathrm{C}=\mathrm{O})$ and $3315(\mathrm{NH}) ;{ }^{1} \mathrm{H}$ NMR $\left(400 \mathrm{MHz}, \mathrm{CDCl}_{3}\right): \delta_{\mathrm{H}} 1.36\left(3 \mathrm{H}, \mathrm{t},{ }^{3} \mathrm{~J}_{\mathrm{HH}}=7.2 \mathrm{~Hz}, \mathrm{CH}_{3}\right), 1.41(3 \mathrm{H}, \mathrm{t}$, $\left.{ }^{3} \mathrm{~J}_{\mathrm{HH}}=7.2 \mathrm{~Hz}, \mathrm{CH}_{3}\right), 2.04\left(3 \mathrm{H}, \mathrm{s}, \mathrm{CH}_{3}\right), 2.32\left(6 \mathrm{H}, \mathrm{s}, 2 \mathrm{CH}_{3}\right), 4.32\left(2 \mathrm{H}, \mathrm{q},{ }^{3} \mathrm{~J}_{\mathrm{HH}}=7.2 \mathrm{~Hz}\right.$, $\left.\mathrm{OCH}_{2}\right), 4.39\left(2 \mathrm{H}, \mathrm{q},{ }^{3} \mathrm{~J}_{\mathrm{HH}}=7.2 \mathrm{~Hz}, \mathrm{OCH}_{2}\right), 6.67(1 \mathrm{H}, \mathrm{s}, \mathrm{CH}=\mathrm{CH}), 7.15(3 \mathrm{H}, \mathrm{m}, \mathrm{Ph}), 7.23(1 \mathrm{H}$, $\left.\mathrm{t},{ }^{3} \mathrm{~J}_{\mathrm{HH}}=7.6 \mathrm{~Hz}, \mathrm{H}_{\mathrm{para}}\right), 7.31\left(2 \mathrm{H}, \mathrm{t},{ }^{3} \mathrm{~J}_{\mathrm{HH}}=7.6 \mathrm{~Hz}, \mathrm{H}_{\text {meta }}\right), 7.34\left(2 \mathrm{H}, \mathrm{d},{ }^{3} \mathrm{~J}_{\mathrm{HH}}=7.6 \mathrm{~Hz}, \mathrm{H}_{\text {ortho }}\right)$, $8.10(1 \mathrm{H}, \mathrm{s}, \mathrm{NH}) ;{ }^{13} \mathrm{C} \mathrm{NMR}\left(400 \mathrm{MHz}, \mathrm{CDCl}_{3}\right): \delta_{\mathrm{C}} 14.13$ and $14.30\left(2 \mathrm{CH}_{3}\right), 14.42\left(\mathrm{CH}_{3}\right)$, $18.51\left(2 \mathrm{CH}_{3}\right), 59.99$ and $61.77\left(2 \mathrm{OCH}_{2}\right), 88.75,114.20,142.58$ and 159.33 (furan), 124.98 and $128.13(\mathrm{C}=\mathrm{C}$ alkene $), 125.83\left(\mathrm{C}_{\text {ortho }}\right), 126.71\left(\mathrm{C}_{\text {para }}\right), 127.10\left(\mathrm{C}_{\text {meta }}\right), 136.99\left(\mathrm{C}_{\mathrm{ipso}}\right)$, $128.44\left(\mathrm{C}_{\text {ortho }}\right), 129.23\left(\mathrm{C}_{\text {para }}\right), 134.20\left(\mathrm{C}_{\text {meta }}\right), 135.17\left(\mathrm{C}_{\text {ipso }}\right), 164.58$ and $165.78(2 \mathrm{CO})$; $\mathrm{MS}$ : $m / z \quad \%): 77(\mathrm{Ph}, 14), 105\left(\mathrm{Ph}\left(\mathrm{CH}_{3}\right)_{2}, 25\right), 121\left(\left(\mathrm{CH}_{3}\right)_{2} \mathrm{PhNH}_{2}, 100\right), 299\left(\mathrm{M}^{+}-2\right.$ $\left.\mathrm{CH}_{3} \mathrm{CH}_{2} \mathrm{COOH}, 19\right), 373\left(\mathrm{M}^{+}-\mathrm{CH}_{3} \mathrm{CH}_{2} \mathrm{COOH}, 4\right), 402\left(\mathrm{M}^{+}-3 \mathrm{CH}_{3}, 30\right), 447\left(\mathrm{M}^{+}, 70\right)$, Anal. Calcd for $\mathrm{C}_{27} \mathrm{H}_{29} \mathrm{NO}_{5}$ (447.50): C, 72.46; H, 6.52; N, 3.13. Found: C, 72.51; H, 6.54; N, 3.14 .

\section{Dimethyl 2-(2,6-Dimethyl-phenylamino)-5-furyl-3,4-furan dicarboxylate (4e):}

Yellow powder, m.p. $140-142{ }^{\circ} \mathrm{C}$, yield $85 \%$, IR $(\mathrm{KBr})\left(v_{\max }{ }^{\prime} \mathrm{cm}^{-1}\right): 1675-1737(\mathrm{C}=\mathrm{O})$ and $3311(\mathrm{NH}) ;{ }^{1} \mathrm{H}$ NMR $\left(400 \mathrm{MHz}, \mathrm{CDCl}_{3}\right): \delta_{\mathrm{H}} 2.30\left(6 \mathrm{H}, \mathrm{s}, 2 \mathrm{CH}_{3}\right), 3.87$ and $3.95(6 \mathrm{H}, 2 \mathrm{~s}, 2$ $\left.\mathrm{OCH}_{3}\right), 6.38\left(1 \mathrm{H}, \mathrm{dd},{ }^{3} \mathrm{~J}_{\mathrm{HH}}=3.5 \mathrm{~Hz},{ }^{4} \mathrm{~J}_{\mathrm{HH}}=1.6 \mathrm{~Hz}, \mathrm{CH}\right), 6.46\left(1 \mathrm{H}, \mathrm{d},{ }^{3} \mathrm{~J}_{\mathrm{HH}}=3.5 \mathrm{~Hz}, \mathrm{CH}\right) 7.15$ $(3 \mathrm{H}, \mathrm{m}, \mathrm{Ph}), 7.37\left(1 \mathrm{H}, \mathrm{d},{ }^{3} \mathrm{~J}_{\mathrm{HH}}=1.6 \mathrm{~Hz}, \mathrm{CH}\right) 8.12(1 \mathrm{H}, \mathrm{s}, \mathrm{NH}) ;{ }^{13} \mathrm{C}$ NMR $\left(400 \mathrm{MHz}, \mathrm{CDCl}_{3}\right)$ : $\delta_{\mathrm{C}} 18.47\left(2 \mathrm{CH}_{3}\right), 51.48$ and $52.54\left(2 \mathrm{OCH}_{3}\right), 87.76(\mathrm{CN}=\mathrm{C}), 107.80$ and $111.28(2 \mathrm{CH})$, $112.32(\mathrm{OC}=\mathrm{C}), 127.24$ and $128.47(\mathrm{Ph}), 134.02(\mathrm{C}), 135.31$ and $135.39(\mathrm{Ph}), 142.69(\mathrm{CH})$, $143.76(\mathrm{C}), 159.99(\mathrm{C}), 164.49$ and $165.06(2 \mathrm{CO})$; MS: $m / z(\%): 168\left(\mathrm{M}^{+}-\mathrm{C}_{12} \mathrm{H}_{12} \mathrm{NO}, 85\right)$, $265\left(\mathrm{M}^{+}-\left[\mathrm{COOCH}_{3}+3 \mathrm{CH}_{3}\right], 91\right), 339\left(\mathrm{M}^{+}-2 \mathrm{CH}_{3}, 92\right), 369\left(\mathrm{M}^{+}, 100\right)$, Anal. Calcd for $\mathrm{C}_{20} \mathrm{H}_{19} \mathrm{NO}_{6}$ (369.35): C, 65.03; H, 5.18; N, 3.79. Found: C, 65.09; H, 5.21; N, 3.81 .

\section{Conclusion}

We studied the synthesis of polyfunctionalized furans with longer conjugated aromatic systems that can show chemiluminescence activities by a three component reaction under the neutral conditions. 


\section{References}

1. Ma C and Yang Y, Org Lett., 2005, 7, 1343.

2. Domling A and Ugi I, Angew Chem Int Ed., 2000, 39, 3168-3210.

3. Ugi I, Angew Chem Int Ed., 1982, 21, 810-819.

4. Zargoosh K, Chaichi M J, Asghari S, Qandalee M and Shamsipur M, J Iran Chem Soc., 2010, 7, 376-383.

5. Zargoosh K, Shamsipur M, Qandalee M, Piltan M, Morad L, Spectrochim Acta A, 2011, 81(1), 679-683.

6. Nair V, Vinod A U and Rajesh C, J Org Chem., 2001, 66, 4427-4429.

7. Asghari S and Qandalee M, Acta Chim Slov., 2007, 54, 638-641.

8. Yavari I, Hossaini Z and Sabbaghan M, Mol Divers., 2006, 10, 479-482.

9. Yavari I, Djahaniani H and Nasiri F, Tetrahedron., 2003, 59, 9409-9412.

10. Yavari I, Mokhtarporyani-sanandaj A, Moradi L and Mirzaei A, Tetrahedron., 2008, 64, 5221-5225.

11. Lipshutz B H, Chem Rev., 1986, 86, 795-819.

12. Dillger H J, Fengler G, schumann D and Winter Feldt E, Tetrahedron., 1974, 30, 2561-2564.

13. Junjappa H, Sanena M K, Ramaiah D, Loharay B B, Rath N P and George M V, $J$ Org Chem., 1998, 63, 9801.

14. Nair V, Menon R S, Beneesh P B, Kumar V S and Bindu S, Org Lett., 2004, 6, 767.

15. Winterfeldt E, Angew Chem Int Ed Engl., 1967, 6, 424.

16. Yavari I, Mohtat B and Zare H, Mol Divers., 2006, 10, 247.

17. Nair V, Menon R and Sreekumar V, Pure Appl Chem., 2005, 77, 1191. 


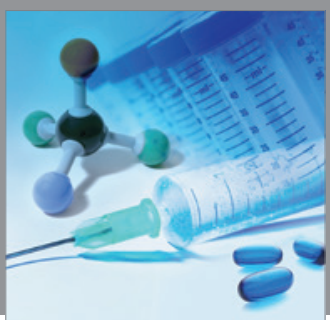

International Journal of

Medicinal Chemistry

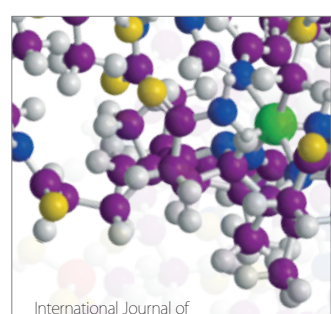

Carbohydrate Chemistry

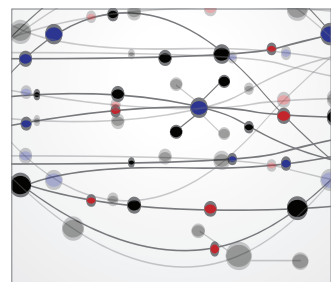

The Scientific World Journal
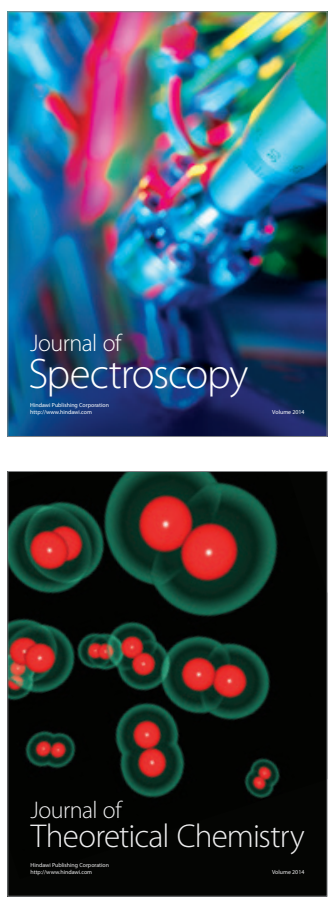
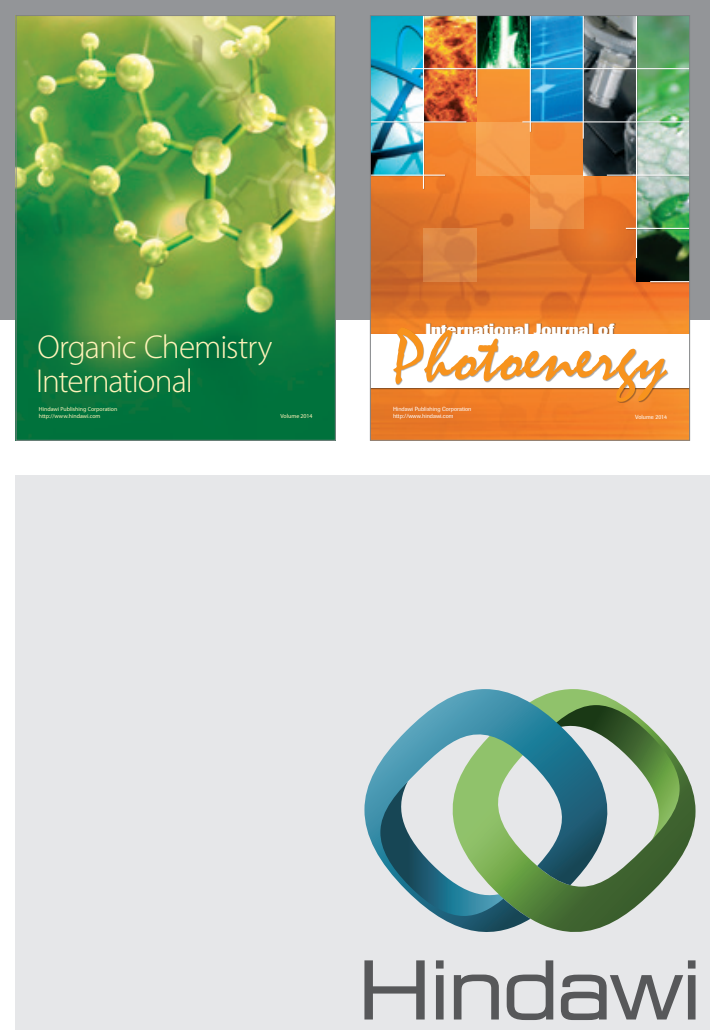

Submit your manuscripts at

http://www.hindawi.com
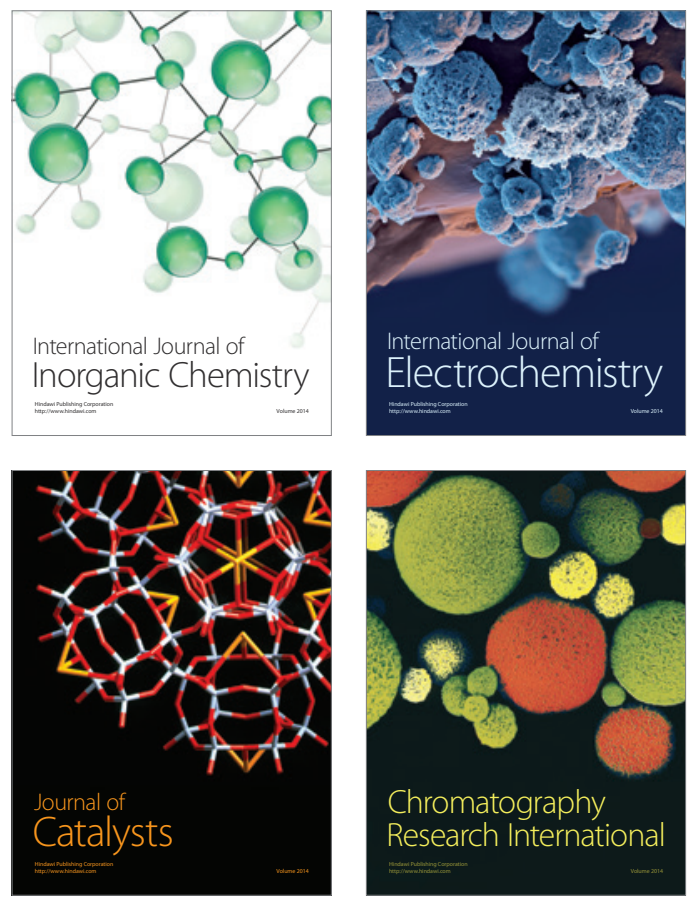
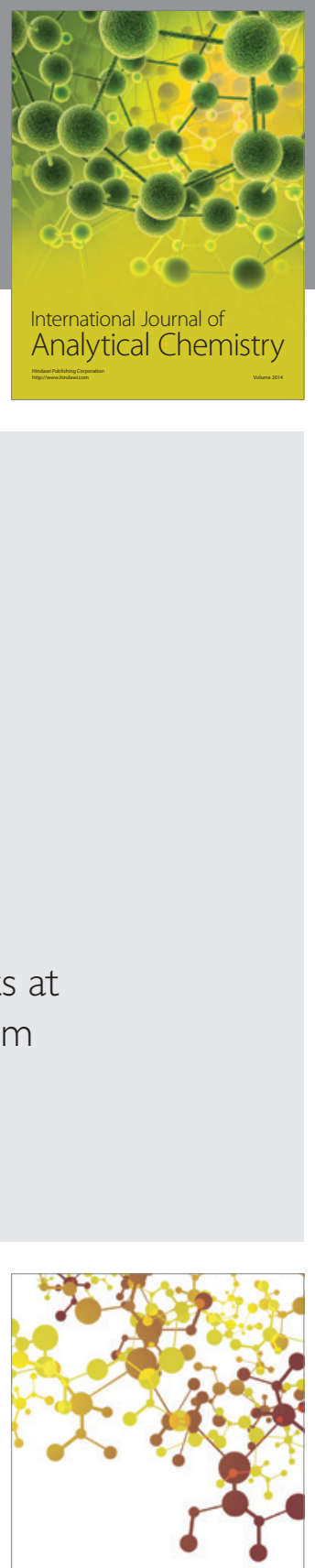

Journal of

Applied Chemistry
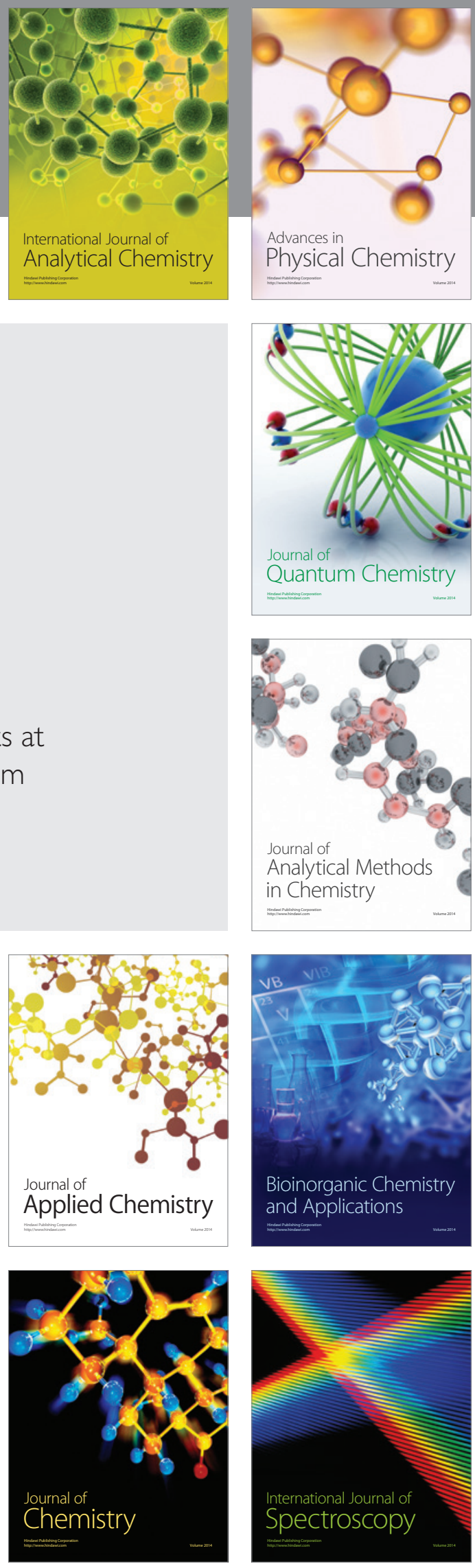